\title{
Role of MRI in the Diagnosis of Meniscal and Ligament Injuries of Knee Joint: A Retrospective Study
}

\author{
Sowmya V ${ }^{1}$, Seetha Pramila V.V. ${ }^{2}$ \\ ${ }^{1}$ Postgraduate, Department of Radiodiagnosis, Raja Rajeshwari Medical College and Hospital, Bengaluru, 2Professor, \\ Department of Radiodiagnosis, Raja Rajeshwari Medical College and Hospital, Bengaluru, India \\ Corresponding author: Seetha Pramila V.V., Professor, Department of Radiodiagnosis, Raja Rajeshwari Medical College and \\ Hospital, Bengaluru, India
}

DOI: http://dx.doi.org/10.21276/ijcmsr.2019.4.4.25

How to cite this article: Sowmya V, Seetha Pramila V.V. Role of MRI in the Diagnosis of Meniscal and Ligament Injuries of Knee Joint: A Retrospective Study. International Journal of Contemporary Medicine Surgery and Radiology. 2019;4(4):D103-D106.

\section{A B S T R A C T}

Introduction: The knee joint is a hinge joint. It has ligamentous and meniscal structures which play an important role in the stability and mobility. Menisci form a cushion and the anterior and posterior cruciate ligaments are important stabilizers of the knee joint.Menisci and cruciate ligaments have slightly different properties and complement each other in function. Study objectives were to describe the MRI appearance of the menisci in their injured state and to describe the MRI appearance of the ligaments in their injured state.

Material and methods: Our study included 100 patients in the age range of 11-60 years who were referred to the Department of Radiology for MRI following injury to the knee. This was a retrospective study and was done over a period of 14 months between May 2018 to June 2019. A detailed history, clinical, and local examination was carried out in all the patients prior to doing the MRI.MRI was carried out on 1.5 Tesla machine and the standard protocol consisted of:

Fat-suppressed PD (TE 45, TR 2800) in axial, sagittal, and coronal planes.

T2W (TE 80, TR 4000) in sagittal plane.

T1W (TE 11, TR 495) in sagittal plane.

Results: In our study, the commonest injury was found to be anterior cruciate ligament injury and tear of posterior horn of medial meniscus. In most of the injuries, there was associated joint effusion and bone contusion. Detailed history, clinical, and local examination of the patient was also helpful in this study.

Conclusions: MRI is a useful non-invasive modality having high diagnostic accuracy making it a very reliable screening test for diagnosing menisci and ligament injuries of knee joint.

Keywords: MRI in the Diagnosis, Meniscal and Ligament Injuries, Knee Joint

\section{INTRODUCTION}

Knee injuries represent roughly $6 \%$ of all acute injuries treated at Emergency Department and between 27\% and $48 \%$ of these have been reported to be sports- related. ${ }^{1,2}$ History taking regarding mechanism of knee injury gives a vital clue to the internal derangements of knee joint. Hyperextension with an audible pop suggests a likely diagnosis of anterior cruciate ligament (ACL) tear. Clinical examination is very important for the diagnosis of ligament injury, but painful stress examinations are not always accurate in the acute stage of the injury. Therefore, Magnetic Resonance Imaging (MRI) is indicated for early diagnosis of the acutely injured knee. ${ }^{2}$ Of the 100 patients, 62 were male, and 38 were females. The average age was 27 years (range: 15 to 60 years).

MRI has been proved to be the choice to evaluate the status of the ACL and other associated structures in the knee. Sagittal MR images have been commonly used in the evaluation of the ACL. ${ }^{1}$ Multiplanar imaging can readily demonstrate meniscal, ligamentous, and bone marrow injuries.

The ACL is injured in field sports, skiing, motor vehicle accidents, and falls. Injuries most commonly occur from actions involving deceleration, twisting, or jumping. Because of the morbidity associated with the ACL-deficient knee, accurate diagnosis of ACL injury is essential for appropriate patient care. ${ }^{1}$ The imaging diagnosis should be based on primary signs, although the secondary signs are sometimes useful, particularly in chronic cases. The detection of associated injuries of other structures of the knee, including menisci, collateral ligaments, cartilage, and bone, are also important.

Accurate interpretation of the MRI examination requires a meticulous MRI technique, knowledge of the imaging anatomy, and understanding of the lesion appearance. This study reviews the MRI appearance of normal and injured cruciate ligaments. Mechanisms of injury, primary and secondary MRI signs, and associated abnormalities are discussed. ${ }^{3}$ 
In our study, we discussed the normal anatomy of knee in MRI and classical features of meniscal, cruciate and collateral ligament injuries of knee joint.

\section{MATERIAL AND METHODS}

Our study included 100 patients in the age range of 1160 years who were referred to Department of Radiology with a history of trauma to the knee. This study was done between May 2018 to June 2019. The type of study done is observational study.

\section{Patient preparation and positioning}

- Written consent taken

- Procedure explained

- Patients instructed to lie still

- Patient should be supine with feet first

- Position the knee coil and then immobilise with cushions

\section{MRI knee protocol}

The three plane localiser is used to plan the sequence. T 1 weighted scan are included. The axial plane slice is then planned on the coronal plane, the position block is parallel to medial and lateral femoral condyle. Slices should be in an angle in sagittal plane (perpendicular to the line of femur and tibia).Coronal and sagittal plane is planned accordingly. The sequences to be included are;

- PD FS Axial,

- PD FS Coronal,

- T1 TSE Sagittal and

- T2 STIR Sagittal.

\section{Parameters}

TR:4000, TE:15, SLICE:3MM, PHASE:R>L, MATRIX:26X256, FOV:150-160

\section{Selection Criteria}

\section{Inclusion criterion}

- Patients referred with history of knee injury.

\section{Exclusion criteria}

- Ferromagnetic implants

- Cardiac pacemakers

- Cochlear implants

\section{RESULTS}

This was a retrospective study of 14 months duration, done

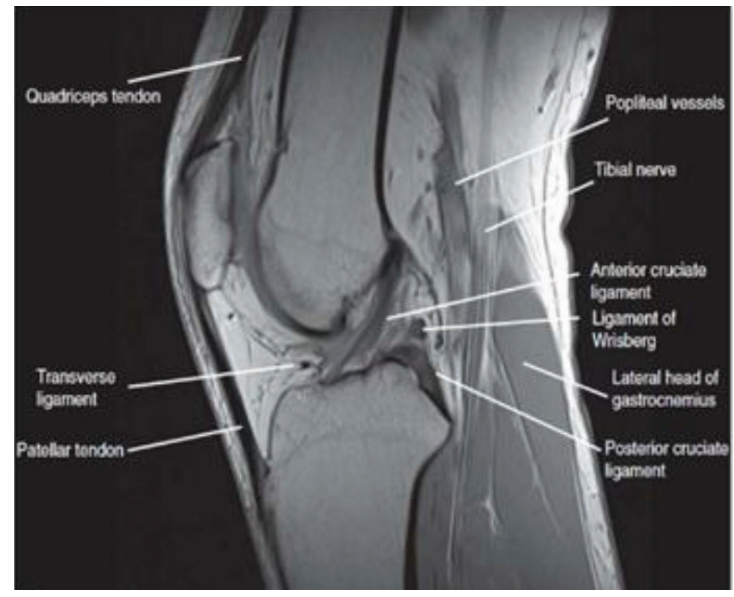

Figure-1: Sagittal(PD) at Department of Radiodiagnosis, Raja Rajeshwari Medical college, Bengaluru. The main aim was to evaluate MRI features in all types of knee injuries. A total of 100 patients with history of injury to knee joint who were referred for MRI scan of knee joint were studied. Majority of the patients were male (62\%) and (38\%) patients were female with the male to female ratio is $4: 1$. The commonest age group was 21 to 30 years for both males and females with mean age of 31.67 years for male and 35.1 years for female. Of the 100 patients, 62 were male, and 38 were females. The average age was 27 years (range: 15 to 60 ).

In our study the most commonly injured ligament was
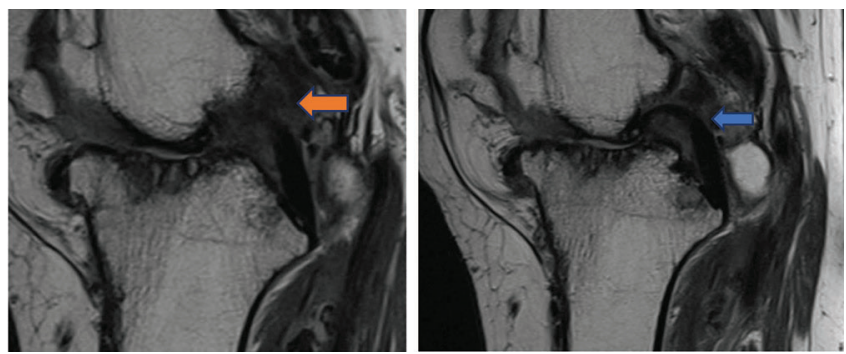

Figure-2: Subsequent sagittal PD MR image confirms a complete tear of the ACL causing buckling of PCL(arrow).

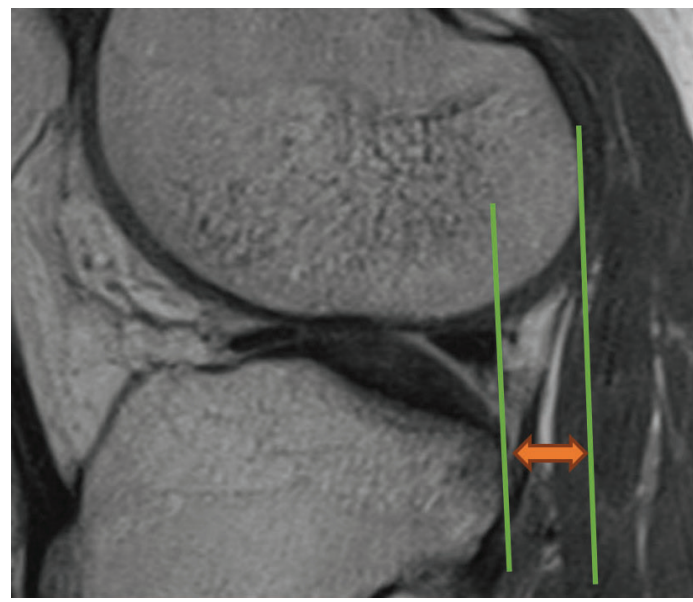

Figure-3: Sagittal PD weighted shows anterior tibial translation (indirect sign) i.e, the two parallel lines drawn from the posterior aspects of the lateral femoral condyle and lateral tibial condyle (it is $7 \mathrm{~mm}$ )

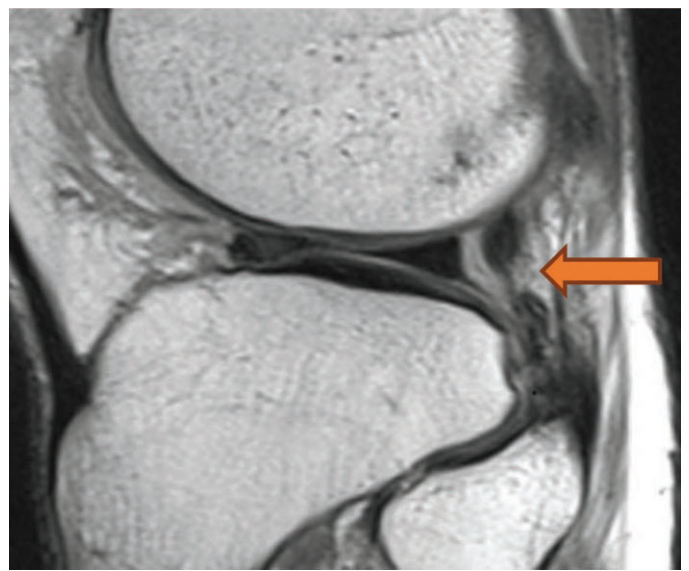

Figure-4: Sagittal PD MR image shows grade III signal intensity change in anterior horn of medial meniscus 
anterior cruciate ligament i.e., there were 24 complete tears and 21 partial tears. Few ACL were bulky due to mucoid degeneration and oedema. Posterior cruciate ligament tear (Total 7 in number) were minimum when compared to ACL tear. Complete PCL were 5 and partial tear of PCL was 2. Most of the injuries were associated with mild -moderate joint effusion. From the present study, it was noted that Magnetic Resonance Imaging is most comprehensive, noninvasive and safe modality in radiological evaluation of knee injury.

In meniscal tears, there were 18 medial meniscal tear and 17 lateral meniscal tears. Medial meniscal tears are maximum with posterior horn involvement. Bucket handle tears were noted among these. Few showed meniscal extrusion also. Whereas in lateral meniscus grade III signal change was more in posterior horn compared to anterior horn of lateral meniscus.

\section{DISCUSSION}

\section{Anatomy}

In the sagittal plane(PD), the primary checklist structures are (1) the medial and lateral menisci, (2) the chondral surfaces of the medial and lateral compartment, (3) the trochlear groove cartilage, and (4) the anterior and posterior cruciate ligaments. Other structures to be observed in the sagittal plane are (5) the posteromedial and posterolateral corners, (6) the patellar and quadriceps tendons, (7) subchondral bone and marrow, (8) joint fluid/effusion, and, (9) Hoffa's fat pad. The tibiofibular joint, collateral ligaments, lateral tendons, and patellar facet articular cartilage may also be evaluated (Figure 1 and 2).

In the coronal plane, the primary checklist structures are (1) the collateral ligaments. Additional structures to be examined include (2) the anterior and posterior cruciate ligaments, (3) the medial and lateral menisci, (4) articular cartilage, (5) osseous structures, and (6) the iliotibial tract.

In the axial plane, the primary checklist structures to be evaluated are (1) the cartilage covering the articular surfaces of the patella (patellofemoral compartment). Additional structures to be evaluated include (2) the intercondylar notch, (3) the menisci, (4) collateral ligaments, and (5) joint fluid/effusion.

Axial images are used to confirm pathology in ligaments, tendons, and muscles that are oriented nearly 90 to the axial plane (including the collateral ligaments and patellar tendon). In addition, specific morphologic changes in meniscal tears are confirmed on axial plane images. The proximal attachment of the ACL to the side wall of the lateral femoral condyle is also directly visualized. Joint effusions and popliteal cysts can be quantified and characterized on axial plane imaging.

\section{Criteria for Meniscal Tears}

There are three basic MR criteria of meniscal tears: a)high intrameniscal signal extending to at least one articular surface (b)which should be seen in at least two slices called two slice touch rule (do not have to be contiguous, e.g. sagittal and coronal slices) c)distortion of the normal meniscal morphology if no prior surgery. ${ }^{5}$

The grading system used for meniscal signal changes are described as: Grade I signal—globular signal intensity within the meniscus not contiguous with the articular surface. Grade II signal - linear signal intensity within the meniscus not contiguous with the articular surface. Grade III signallinear signal intensity within the meniscus extending to the articular surface.

The criteria for normal anterior cruciate ligament are: a) Fibers to be continuous from all the way from the tibia to the femur. b)Fiber orientation to be as steep or steeper than the intercondylar roof. The criteria for normal posterior cruciate ligament are it has to be more linear, ACL has helically oriented fibers and are more uniformly hypointense than ACL.PCL fibers prevent posterior translation of tibia \& anterior translation of femur.PCL fibers are larger and twice as strong as ACL.

Lee et.al have demonstrated that significant bowing of PCL is a sensitive indicator of ACL tear.

The primary signs of ligament tear are the fibers are discontinued seen mainly on T2, show abnormal orientation and there is bone edema noted in fat suppressed sequences. Traumatic injuries of the MCL are common and range from intrasubstance tears (grade 1 sprain) to incomplete tears (grade 2 sprain) to complete disruption (grade 3 sprain).

The primary signs of ACL tear are ${ }^{9,8}$

- Ligament discontinuity of ACL

- Diffuse or focal abnormal signal intensity (coronal images should be used in conjunction with sagittal images to compensate for segmental visualization in the sagittal plane),

- Abnormal orientation or ligament course (abnormal blumensaat angle),

- A mass-like appearance in the expected location of the ACL,

- Non-visualization of the ACL.

The secondary signs and other predictors of ACL tear are ${ }^{10}$

- Osseous contusions in the lateral compartment (posterolateral tibial plateau is most specific), contusion at mid portion of lateral femoral condyle and posterior portion of lateral tibial plateau are termed as kissing contusions representing edema and haemorrhages due to impaction microfactures of trabecula,

- Buckling of posterior cruciate ligament,

- Uncovered posterior horn of lateral meniscus - A vertical line drawn tangentially to the posterior most margin of the lateral tibial plateau intersects the posterior horn of lateral meniscus,

- Anterior tibial displacement (assessed in the lateral aspect of the lateral compartment) - The distance between two parallel lines drawn tangential to the posterior lateral Femoral condyle and posterior lateral Tibial plateau is more than $5 \mathrm{~mm}$.

The mucoid degeneration of ACL is a rare cause of knee pain which predominantly affects elderly women.

\section{CONCLUSION}

MRI plays a primary and crucial role in ACL injuries and its management by allowing confident diagnosis of whether there is an ACL injury or not, especially in patients with equivocal physical examination and also provides additional information regarding the injuries of associated ligaments. 


\section{REFERENCES}

1. E M Remeret.al Anterior cruciate ligament injury: MR imaging diagnosis and patterns of injury Radiographics. 1992;12:901-15.

2. Frobell RB et al., Acute rotational trauma to the knee: Poor agreement between clinical assessment and magnetic resonance imaging findings. Scand J Med Sci Sports 2007;17:109-14.

3. C. K. Kamet al., Magnetic Resonance Imaging of Cruciate Ligament Injuries of the Knee 2010:61;80e89.

4. Rajesh Umap, Bijpuriya Anurag, Sachin Bagale, Navid Shattari. Evaluation of traumatic knee joint injuries with MRI. International Journal of Contemporary Medicine Surgery and Radiology. 2018;3:C77-C81.

5. Stoller et al., Magnetic Resonance Imaging in orthopaedics and sports medicine, $3^{\text {rd }}$ edition $2^{\text {nd }}$ volumeCopyright (C) 2007 by Lippincott Williams \& Wilkins.

6. Ali M. Naraghi, et.al Imaging of Athletic Injuries of Knee Ligaments and Menisci: Sports Imaging Series, September 2016

7. Jeffrey $\mathrm{S}$ prince et.al MRI of anterior cruciate ligament injuries and associated findings in paediatric knee: changes with skeletal maturation. American Journal of Roentgenology. 2005;185: 756-762.

8. Nilesh $\mathrm{H}$ et al. MR imaging of anterior cruciate ligament injuries. Int J Res Med Sci. 2017;5:4980-4987

9. Barry KP, Mesgarzadeh M, Triolo J, et al. Accuracy of MRI patterns in evaluating anterior cruciate ligament tears. Skeletal Radiol 1996;25:365-70.

10. Robertson PL, Schweitzer ME, Bartolozzi AR, et al. Anterior cruciate ligament tears: evaluation of multiple signs with MR imaging. Radiology 1994; 193:829-34

11. Gentili A, Seeger LL, Yao L, et al. Anterior cruciate ligament tear: indirect signs at MR imaging. Radiology 1994;193:835-40.

12. Bolog NV, Andreisek G, Ulbrich EJ. MRI of the Knee: A Guide to Evaluation and Reporting. Springer; 2015.

13. Barry KP, Mesgarzadeh M, Triolo J, et al. Accuracy of MRI patterns in evaluating anterior cruciate ligament tears. Skeletal Radiol 1996;25:365-70.

14. Bergin D, et al. Anterior cruciate ligament ganglia and mucoid degeneration: coexistence and clinical correlation.AJR Am J Roentgenol. 2004;182: 1283-7.

Source of Support: Nil; Conflict of Interest: None

Submitted: 09-10-2019; Accepted: 04-10-2019; Published online: 30-11-2019 